\title{
Temperature-dependent energy levels and the second law formulation for engineering devices
}

\section{Takuya Yamano}

Department of Mathematics and Physics, Faculty of Science, Kanagawa University, 2946, 6-233

Tsuchiya, Hiratsuka, Kanagawa 259-1293, Japan; E-Mail: yamano@amy.hi-ho.ne.jp, Tel./Fax: +81 45 4728796.

Published: 13 November 2015

\begin{abstract}
We shall give an overview, within a scope of the author's knowledge, on fundamental importance of the temperature dependency of energy levels in materials in quantifying heat dissipated in thermodynamically operated devices. After a brief review of the relevant studies of temperature dependency in semiconductors, we point out that entropy produced or heat dissipated in thermoelectric devices based on thermoelectric effects such as Peltier, Seebeck and Thomson can be reformulated by inclusion of the effect of temperature-dependent energy levels in materials (semiconductors). Statistical mechanics consideration for the origin of the temperature dependent energy levels is also given based on a general ensemble theory.
\end{abstract}

Keywords: temperature-dependent energy levels; second law of thermodynamics; irreversibility; thermoelectric device

PACS classifications: 05.70.-a, 05.70.Ln

\section{Introduction}

The population in the world is estimated to reach 10 billion in 2050 and the energy needs will be more than 33.2[TW] (the figure was 13.6[TW] in 2002 and 17.8[TW] in 2012) [1]. The decarbonization, low carbon energy supplies, and low carbon industry processes in our society are inevitable. Concurrently, the reuse of waste heat from vehicles, industrial factories and other human activities is becoming a significant and a serious issue over the globe than ever before for our sustainable society. In this sense, thermoelectric generation technology because of the environmental merit is proportionally getting a necessary and a promising research direction [2]. In a form of an overview as a keynote presentation 
of the 2nd International Electronic Conference on Entropy and its Applications (section Physics and Engineering), this article deals with a topic that physics meets engineering in a fundamental level and we intend to get an insight into the interface via the entropy conception. The present author is neither an engineer nor an expert in thermoelectricity, however, it will be a benefit by considering this problem from a possible angle that has never been challenged: the dissipated work observed in a process of thermoelectric operation or the second law of thermodynamics formulation in which the effect of the temperature-dependent energy levels of thermodelectric materials is taken into consideration. In general, the properties of materials depend on temperature. Accordingly, their electrical and thermal conductivity varies with temperature, the influences of which can be very important in thermoelectric generation [3].

The organization of this article is as follows. We shall first attempt to summarize the past studies on temperature dependency in semiconductor and we point out why a new expression of irreversibility processes (i.e, the second law of thermodynamics) seems necessary for a legitimate description of entropy production in thermoelectric devices. In Section 3, we consider the temperature-dependent energy levels from the general statistical ensemble theory. The literature of thermoelectricity is vast and has a long tradition. Therefore, the comprehensive overview is beyond the author's ability and is not the present purpose. We limit ourselves to cite relevant and major references. Also, an overview of the state-of-the-art and recent advances in thermoelectric materials and their properties is not included. In Section 4, we overview the extended expressions of the second law. Some new results are also presented in Sec. 4.4. The last section provides a summary statement.

\section{Thermoelectric phenomena as irreversible process}

Temperature differences between two contacted conductive materials can induce heat flow; it is observed by measuring voltage and current. Conversely, electrically induced heat flow causes temperature difference $(d T)$ between them. One of the striking irreversible processes is these thermoelectric phenomena known as the Seebeck and the Peltier effects observed in semiconductor materials [4]. When the total current is $I$, the Seebeck coefficient is defined by the relation $S=$ $d V /\left.d T\right|_{I=0}$, where $d V$ is the Seebeck elective motive force across the device boundary. On the other hand, when the induced current is $I_{i d}$, the Peltier coefficient is defined as $\Pi=\dot{Q} /\left.I_{i d}\right|_{d T=0}$, where $\dot{Q}$ is the total heat flux. The relation between the two coefficients, i.e., $\Pi=S T$ (the second Kelvin relation) was obtained in 1930's by the second law of thermodynamics by Thomson [4]. The measurement technique of the Seebeck coefficient and other thermoelectric quantities are described in detail, e.g., in chapter 8 of Ref. [3].

Thermoelectricity has a long tradition since its discovery by Thomas J. Seebeck, Jean C. A. Peltier and William Thomson (Lord Kelvin) [5-7]. It was already referred to as revival more than half a century ago [8] (see [9] for a historical overview.). However, it is now active discipline as a fundamental physics as well as an engineering pursuit. In this article, we shall have interests in thermoelectricity in terms of the manifestation of the second law of thermodynamics. As Tolman and Fine [10] had recognized, a measure of the total irreversibility occurring in the thermoelectric process can be quantified by the 
entropy production within the system. In this line, we focus on the second law within a thermodynamic device.

\subsection{Evidences: Temperature-dependency in semiconductors}

The bandgap in semiconductors dependents on temperature (see e.g. [3] for the introduction from an engineering aspect). In semiconductor materials, the electronic energy levels of a charge carrier (electron or hole) shift with temperature as a result of interaction with lattice vibrations, i.e. the electron-phonon interaction [11]. In addition, there is an effect of the lattice expansion/contraction depending on the temperature. Accordingly, the temperature dependence of energy gap $E_{g}(T)$ between the conduction and the valence bands becomes particularly important. The phenomenological relation at temperature $T$ has been given by Varshni [12] in the literature:

$$
E_{g}(T)=E_{0}-\frac{\alpha T^{2}}{T+\beta},
$$

where $\alpha$ and $\beta$ are constants that are specific to materials. The $E_{0}$ is its value at the zero temperature. A recent refined formulation is given by using the Bose-Einstein statistical factor and prominent transverse-acoustic and transverse-optical bands in the measured or calculated phonon density of states ([13] and references therein).

Among three temperature ranges from low to high, i.e., the ionization, the intrinsic, and the extrinsic range in doped materials, it is known that carrier densities in the intrinsic range strongly depend on temperature (proportional to $T^{3 / 2} \exp \left(-E_{0} / 2 k T\right)$ ) [14]. On the other hand, they are constant over a wide range of temperature in the extrinsic range. The carrier mobility $\mu$ of materials is also highly temperature dependent. As temperature increases, mobility decreases as lattice vibrations cause carriers more scatter. This effect is proportional to $T^{-3 / 2}$ at high temperatures but it becomes linearly proportional to $T$ at low temperatures [14].

In addition to the seek for the appropriate gap formulation that includes temperature effects, the studies of temperature dependences of electronic energy levels in an understanding of electronic properties of solids have been one of the old important topics (e.g. $[15,16])$. Also, the treatment in the framework of statistical mechanics has been addressed [15,17]. A recent consideration is presented in Ref. [18]. Experimentally, the evidence of temperature-dependent energy levels in conductivity and mobility has been reported [19]. Its direct effect on the Peltier heat, an isothermal thermodynamic quantity supplied by a move of a charge carrier into a material, was discussed based on the Helmholtz free energy more than 30 years ago ([20-22] and references cited therein). It should be noted that these materials are used as thermoelectric heater, cooler, and generator in practical applications.

We shall overview the results reported in Refs. [20-22] here to validate our viewpoint of improving or refining the expression of the second law of thermodynamics. Energy levels in materials change with temperature dominantly by an interaction of electrons with lattice vibrations [19]. The thermal vibration of lattice affects frequency of collisions and causes a broadening of electron energy levels resulting in 
reducing the bandgap. It affects significantly the Peltier heat device. The Peltier heat associated with a carrier in the $k$ th electronic state is given as

$$
\Pi_{k}=E_{k}^{Q}-\mu
$$

where $E_{k}^{Q}$ is the quasiparticle energy defined as $E_{k}^{\prime}-T \partial E_{k}^{\prime} / \partial T$ with an effective electronic energy $E_{k}^{\prime}$ and $\mu$ is the chemical potential. The weighted average of each quantity with respect to the contribution of the $k$ th level to the electric conductivity $\sigma_{k}$ reads

$$
\Pi=\frac{\sum_{k} \Pi_{k} \sigma_{k}}{\sum_{k} \sigma_{k}}=\frac{\sum_{k} E_{k}^{\prime} \sigma_{k}}{\sum_{k} \sigma_{k}}-T \frac{\sum_{k} \frac{\partial E_{k}^{\prime}}{\partial T} \sigma_{k}}{\sum_{k} \sigma_{k}}-\mu .
$$

This quantity can be comparable to the expression derived from a model of phonon occupation [20]; $\Pi=$ $E_{e}+\left(A k_{B}-\gamma\right) T-\mu$, where $E_{e}$ is the edge of the conduction band of the semiconductor and the constant $A$ is a range of energies above the edge, and $k_{B}$ is the Boltzmann constant. The positive coefficient $\gamma$ characterizes the shift of the electric energy levels. Therefore, we have a formal correspondence as the source of temperature dependence of the Peltier heat; that is, the quantity $\left\langle\partial E_{k}^{\prime} / \partial T\right\rangle$ is identified with the coefficient $\gamma-A k_{B}$, which is a positive value. On the other hand, the analysis of the thermoelectric effects in terms of three independent kinetic coefficients $L_{11}, L_{12}$, and $L_{22}$ with the Onsager reciprocity relation has been presented [4,9], in which the change of entropy $\Delta S$ of the material when an electron is added to it is given by $L_{21} /\left(T L_{11}\right)$. As an averaged property, this entropy transported per carrier has a direct link to the Seebeck coefficient, and in this sense, it is a fundamental quantity. The Peltier heat is thus given as $\Pi=T \Delta S$. By combining this relation with the expression of $\Pi$ i.e., Eq. (3) together, we have an interpretation:

$$
\left\langle\frac{\partial E_{k}^{\prime}}{\partial T}\right\rangle=\frac{\left\langle E_{k}^{\prime}\right\rangle-\mu}{T}-\Delta S
$$

If the effective energy level does not depend on the temperature, i.e., the quantity $\left\langle\partial E_{k}^{\prime} / \partial T\right\rangle$ vanishes, we reduce to the usual formula for the Peltier heat; $\left\langle E_{k}^{\prime}\right\rangle-\mu$. Summarizing the point up to here, the extra temperature-dependent term appearing in a form of heat is attributed to the linear coefficient of the temperature in the Peltier heat formula and it is also caused by entropy change by the addition of an electron in the material.

In addition to the above evidences for necessity of the inclusion of temperature dependent energy levels into consideration, we mention a recent work on the solar cell material, $\mathrm{MAPBI}_{3}$ (methylammonium lead iodide) perovskite, whose photovoltaic conversion efficiency is getting record high recently. The perovskite material is an emerging and next-generation material for efficient photovoltaic cells (e.g. [23] and cited therein). Interestingly, the change in the energy values of the valence band maximum and the conduction band minimum of $\mathrm{MAPBI}_{3}$ show the downward shift and they are attributed to the change in the bandgap. The shifted values are recently measured as $110 \mathrm{meV}$ and $77 \mathrm{meV}$ respectively as temperature increases from $28^{\circ} \mathrm{C}$ to $85^{\circ} \mathrm{C}$ [24]. 


\subsection{Thermoelectricity as a heat engine}

A concise review on thermoelectric phenomena and its analogy to a thermodynamic engine is presented in Ref. [9]. We only mention the structural setting for the purpose of the subsequent sections. A sample of thermoelectric material, inside which the Fermi gas (electrons) is confined as a working fluid instead of a molecule gas in a steam engine. It is maintained at a higher temperature at one end and the other side at a lower one. We usually expect extracting a work from the device in a suitable condition.

\section{Origin of temperature dependency in statistical mechanics}

We present the origin of temperature dependent energy levels in statistical mechanics by considering a general statistical ensembles, in which the canonical ensemble is only a particular case. The author owes greatly to Landsberg [25] for this section.

\subsection{Gibbs-Helmholtz pair in general context}

The use of ensembles in statistical mechanics suggests the possibility of writing for a probability

$$
P_{l}=\frac{g\left(\eta_{l}\right)}{\xi_{T}}, \quad \xi_{T} \equiv \sum_{l} g\left(\eta_{l}\right), \quad(1 \leq l \leq n),
$$

where the arbitrary function $g$ should be specified by the key properties of ensembles. In Eq. (5), $g\left(\eta_{l}\right)$ can depend on an energy $E_{l}$ divided by $k_{B} T$. This energy could be an enthalpy for example. Thus $E_{l} \equiv k_{B} T \xi_{T}$ can be a temperature-dependent quantity, and the ensemble specified by Eq. (5) is contracted.

One can extract analogues of average energies and free energies from Eq. (5) by differentiating with respect to $T$ with a set of thermodynamic variables $\{x\}$ to be constant. For examples, $\{x\}$ can stand for volume and number of particles $(v, N)$ for canonical ensembles or pressure and number of particles $(p, N)$ for pressure ensembles. One finds

$$
k_{B} T^{2}\left(\frac{\partial \ln P_{l}}{\partial T}\right)_{\{x\}}=k_{B} T^{2}\left(\frac{\partial \ln g\left(\eta_{l}\right)}{\partial T}\right)_{\{x\}}-k_{B} T^{2}\left(\frac{\partial \ln \xi_{T}}{\partial T}\right)_{\{x\}} .
$$

Multiplying Eq. (6) by $P_{l}$ and summing over $l$, we find

$$
k_{B} T^{2} \sum_{l} P_{l}\left(\frac{\partial \ln g\left(\eta_{l}\right)}{\partial T}\right)_{\{x\}}=k_{B} T^{2}\left(\frac{\partial \ln \xi_{T}}{\partial T}\right)_{\{x\}} \equiv \epsilon_{\{x\}},
$$

where we used that the last term in Eq. (6) does not depend on these parameter values,

$$
\sum_{l} P_{l}\left(\frac{\partial \ln P_{l}}{\partial T}\right)_{\{x\}}=\sum_{l}\left(\frac{\partial P_{l}}{\partial T}\right)_{\{x\}}=\frac{\partial}{\partial T}\left[\sum_{l} P_{l}\right]=0 .
$$

The canonical ensemble result $U=k_{B} T^{2}(\partial \ln Z / \partial T)_{V, N}$ makes one think of $\epsilon_{\{x\}}$ as a generalized average energy. Furthermore, one can write Eq. (7) differently:

$$
k_{B} T^{2} \sum_{l} P_{l}\left(\frac{\partial \ln g\left(\eta_{l}\right)}{\partial T}\right)_{\{x\}}=\tilde{\epsilon}_{\{x\}}-T\left(\frac{\partial \tilde{\epsilon}_{\{x\}}}{\partial T}\right)_{\{x\}}=\epsilon_{\{x\}}
$$


where we have introduced

$$
\tilde{\epsilon}_{\{x\}} \equiv-k_{B} T \ln \xi_{T} .
$$

The suffix $\{x\}$ on the left is intended as an indication that $\tilde{\epsilon}_{\{x\}}$ is conjugate to $\epsilon_{\{x\}}$. The canonical ensemble result $F=-k_{B} T \ln Z$ makes one think of $\tilde{\epsilon}_{\{x\}}$ as a free energy. Based purely on Eq. (5), we have therefore obtained a pair of quantities $\epsilon_{\{x\}}$ and $\tilde{\epsilon}_{\{x\}}$ which are related by the Gibbs-Helmholtz $(\mathrm{GH})$ relation Eq. (8). Typical pairs are not only internal energy $U$ and Helmholtz free energy $F$ with $\{x\}=(V, N)$, but also enthalpy $H$ and Gibbs free energy $G$ with $\{x\}=(p, N)$, since

$$
F-T\left(\frac{\partial F}{\partial T}\right)_{V, N}=U, \quad G-T\left(\frac{\partial G}{\partial T}\right)_{p, N}=H .
$$

Let a heat capacity at constant $x$ be given such that

$$
\epsilon_{\{x\}}(T)=\int_{0}^{T} C_{\{x\}}(\tau) d \tau .
$$

Then if $\left(\tilde{\epsilon}_{\{x\}}, \epsilon_{\{x\}}\right)$ is a GH pair in the sense of Eq. (8) or Eq. (10), we have the following facts

(i) Integrant of the GH relation:

$$
\tilde{\epsilon}_{\{x\}}(T)=-T \int_{0}^{T} \frac{\epsilon_{\{x\}}(\tau)}{\tau^{2}} d \tau=-T \int_{0}^{T} \frac{d \tau}{\tau^{2}} \int_{0}^{\tau} C_{\{x\}}(y) d y .
$$

(ii)

$$
\left[\left(\frac{\partial \tilde{\epsilon}_{\{x\}}}{\partial T}\right)_{\{x\}},\left(\frac{\partial\left(\tilde{\epsilon}_{\{x\}}+\epsilon_{\{x\}}\right)}{\partial T}\right)_{\{x\}}\right]
$$

is also a GH pair.

The fact (ii) is easily seen by the relation $\partial^{2} \tilde{\epsilon}_{\{x\}} / \partial T^{2}=-T^{-1} \partial \epsilon_{\{x\}}(T) / \partial T$ obtained from Eq. (11) and since the GH relation Eq. (8) for $\left(\partial \tilde{\epsilon}_{\{x\}} / \partial T\right)_{\{x\}}$, instead of $\tilde{\epsilon}_{\{x\}}$, is

$$
\left(\frac{\partial \tilde{\epsilon}_{\{x\}}}{\partial T}\right)_{\{x\}}-T\left(\frac{\partial^{2} \tilde{\epsilon}_{\{x\}}}{\partial T^{2}}\right)_{\{x\}}=\left(\frac{\partial \tilde{\epsilon}_{\{x\}}}{\partial T}\right)_{\{x\}}+\left(\frac{\partial \epsilon_{\{x\}}}{\partial T}\right)_{\{x\}} .
$$

The right hand side replaces $\epsilon_{\{x\}}$ of Eq. (8). It is obtained by using the fact that differentiation of $T\left(\partial \tilde{\epsilon}_{x} / \partial T\right)_{\{x\}}=\tilde{\epsilon}_{\{x\}}-\epsilon_{\{x\}}$ yields

$$
T\left(\frac{\partial^{2} \tilde{\epsilon}_{\{x\}}}{\partial T^{2}}\right)_{\{x\}}=-\left(\frac{\partial \epsilon_{\{x\}}}{\partial T}\right)_{\{x\}} .
$$

Observe that by virtue of Eq. (8) and Eq. (9)

$$
-\left(\frac{\partial \tilde{\epsilon}_{\{x\}}}{\partial T}\right)_{\{x\}}=k_{B} \ln \xi_{T}+\frac{\epsilon_{\{x\}}}{T} .
$$

We know that, for $\{x\}=(v, N)$ and $(p, N), \tilde{\epsilon}_{\{x\}}$ is $F$ and $G$ respectively, and from normal thermodynamics that the entropy satisfies

$$
S=-\left(\frac{\partial F}{\partial T}\right)_{v, N}=-\left(\frac{\partial G}{\partial T}\right)_{p, N} .
$$

Thus Eq. (13) gives possible entropy definitions in our general frame when the variable $\{x\}$ are kept constant. 


\subsection{Entropy and temperature-dependent energies}

An entropy-type expression is, using Eq. (5)

$$
-k_{B} \sum_{l} P_{l} \ln P_{l}=-k_{B} \sum_{l} P_{l} \ln g\left(\eta_{l}\right)+k_{B} \ln \xi_{T} .
$$

Noting that in standard thermodynamics,

$$
S=\frac{U-F}{T}=\frac{H-G}{T},
$$

we adopt instead of Eq. (15) the definition (which agrees with Eq. (13))

$$
S \equiv \frac{\epsilon_{\{x\}}-\tilde{\epsilon}_{\{x\}}}{T}=\frac{\epsilon_{\{x\}}}{T}+k_{B} \ln \xi_{T} .
$$

By Eq. (7) together with Eq. (15) and Eq. (8), Eq. (17) yields

$$
\begin{aligned}
S & =k_{B} T \sum_{l} P_{l}\left(\frac{\partial \ln g\left(\eta_{l}\right)}{\partial T}\right)_{\{x\}}-k_{B} \sum_{l} P_{l} \ln P_{l}+k_{B} \sum_{l} P_{l} \ln g\left(\eta_{l}\right) \\
& =-k_{B} \sum_{l} P_{l}\left\{\ln P_{l}-\left[\frac{\partial}{\partial T} T \ln g\left(\eta_{l}\right)\right]_{\{x\}}\right\} .
\end{aligned}
$$

Before discussing Eq. (18) note that Eq. (7) gives

$$
\epsilon_{\{x\}}=-\sum_{l} \frac{d \ln g\left(\eta_{l}\right)}{d \eta_{l}} P_{l}\left[E_{l}-T\left(\frac{\partial E_{l}}{\partial T}\right)_{\{x\}}\right] .
$$

A possibility of finding Eq. (19) in the standard form

$$
\epsilon_{\{x\}}=\sum_{l} P_{l} E_{l}
$$

occurs if $g\left(\eta_{l}\right)=\exp \left[-\eta_{l}\right]$ when Eq. (19) is

$$
\epsilon_{\{x\}}=\sum_{l} P_{l}\left[E_{l}-T\left(\frac{\partial E_{l}}{\partial T}\right)_{\{x\}}\right]
$$

and Eq. (18) is

$$
S=-k_{B} \sum_{l} P_{l}\left[\ln P_{l}+\frac{1}{k_{B}}\left(\frac{\partial E_{l}}{\partial T}\right)_{\{x\}}\right] .
$$

Thus the use of the ensemble in general frame with the exponential $g$-function may not lead to the usual expression Eq. (20) for the average energy or for the average enthalpy or for the average of whatever energy $E_{l}$ represents. Nor do we always find the usual expression, given by the left hand side of Eq. (15), of the entropy: There are correction terms $\left\langle\left(\frac{\partial E_{l}}{\partial T}\right)_{\{x\}}\right\rangle$ due to the possible temperature-dependence of the energies $E_{l}$.

Alternatively, one can choose the terms arising from the temperature dependence of $E_{l}$ in Eq. (7) to be eliminated altogether. One then requires the function $g$ to be such that the general equation Eq. (7) reduces to the special form Eq. (20). Thus

$$
k_{B} T^{2}\left(\frac{\partial \ln g\left(\eta_{l}\right)}{\partial T}\right)_{\{x\}}=E_{l},
$$


i.e.

$$
g\left(\eta_{l}\right)=\exp \left[\int_{T_{0}}^{T} \frac{E_{l}}{k_{B} \tau^{2}} d \tau\right] \equiv \exp \left[-\frac{\tilde{E}_{l}}{k_{B} T}\right] .
$$

Here $T_{0}$ is a fixed temperature and the newly introduced quantity $\tilde{E}_{l}$ satisfies

$$
\tilde{E}_{l}-T\left(\frac{\partial \tilde{E}_{l}}{\partial T}\right)_{\{x\}}=E_{l} .
$$

Thus one may also use in the Boltzmann factor quantities $\tilde{E}_{l}$ related to the given temperature-dependent energies $E_{l}$ by a GH relation. If the $E_{l}$ are energies, one may use a free energy in the exponent of the Boltzmann factor; if the $E_{l}$ are enthalpies one may use a Gibbs free energy in the exponent. The exponential form set for $g$ represents a special case. There are clearly other possibilities as well and the thermodynamic structure is preserved [26,27].

More precisely, $E_{l}$ 's are determined by a set of quantum numbers, which specify a state of system and are subdivided into two sets $\{\alpha\}$ and $\left\{\alpha^{\prime}\right\}$, i.e., $E_{l}\left(\{\alpha\},\left\{\alpha^{\prime}\right\}\right)$. The set $\{\alpha\}$ represents the set of interest and its occurrence as a suffix tells one that the form of the function ( $\xi_{T}$ and $P_{l}$ in the above case) depends on the choice of $\{\alpha\}$. Its occurrence as a parameter tells one that the value of the function depends parametrically on the value which the $i$ 's have. The temperature-dependence arises from the summation over the quantum numbers in the irrelevant set of $\left\{\alpha^{\prime}\right\}$. If the set $\left\{\alpha^{\prime}\right\}$ is empty, however, it can represent a normal canonical ensemble in which the $E_{l}(\{\alpha\})$ are solutions of the Schrödinger equation and therefore independent of temperature. These settings become significant when one considers a transition from statistical mechanics to thermodynamics [28].

\section{Improvement of the second law expression}

Having obtained enough justification of considering temperature-dependent energy levels, we are now in a position to think about how it is reflected into the effective description of the second law. The second law has arguably been a central issue in thermodynamics in irreversible nature [29-31]. In spite of these background described in the previous sections, it has only recently attempted to incorporate the dependency of temperature into the heat irreversibly lost in the thermodynamic processes. We particularly have interest in the dissipated heat or entropy production and how it alters the expression of the second law of thermodynamics.

\subsection{Some backgrounds}

It is common and usual to express the Clausius inequality as an equality (i.e., entropy balance equation) in chemical engineering and mechanical engineering literature [32-35] (We summarize the terminology at the end of this section). In fact, Clausius himself has introduced an equality form instead of inequality to evaluate the amount of entropy produced irreversibly in total system [36]:

$$
\Delta S-\int \frac{d Q}{T}=N
$$


where $\Delta S$ here denotes the difference in entropy between initial and final states and $d Q$ denotes heat exchanged. The positive quantity $N$ (due to the very definition of the second law in the Clausius's sense) represents the degree of the entropy production within a system in irreversible processes. It was originally termed as uncompensirte Verwandlung (uncompensated transformation) by him [36-38]. The corresponding explanation can be seen in Section 3.4 of the modern textbook [38,39]). Tolman and Fine [10] have emphasized significance of being able to express it in the form of an equality by including the entropy that produced irreversibly inside the system and have discussed the validity of the expression. They termed it the second law equality.

Shortly thereafter, the local formalism for continuous open systems by Prigogine and Onsager became a well-known formulation [39], in which the change in entropy is attributed to two sources; the entropy change $d_{e} S$ due to exchange of matter and energy through boundaries of a system, and the entropy production within a system $d_{i} S$ by an irreversible process. In this approach, one also leaves behind the original inequality and only needs to consider the equality.

We next mention two recent approaches to the equality form of the second law. The first one is taken by Ben-Amotz and Honig [40,41]. They have presented it as a rectified form by introducing the entropy deficit function $\theta$. It quantifies the total entropy change in both system and heat bath (note that the symbol $\theta$ does not denote the conventional empirical temperature). By this the Clausius inequality becomes an equality (A recent review on this approach can be accessed in Ref. [42]). In this approach to irreversible work, there is no need to invoke and specify thermodynamic forces and dissipation fluxes as per the formalism by Prigogine and Onsager. Furthermore, they combined the net entropy production with a work relation of irreversible processes found by Jarzynski to obtain the rectified form, aiming at experimentally and numerically evaluating the thermodynamic dissipative work. These considerations can offer a litmus test of the validity in the light of advances on RNA pulling experiments, in which the work value can be directly measured in such irreversible molecular processes [43-45].

On the other hand, the authors of Refs. [46,47] have derived a generalized second law in modeling communication channels as a thermal system. They derived the following form: $d S=d Q / T-T^{-1}\langle d E / d T\rangle d T$, where $d Q$ is heat absorbed by the system with temperature $T$ and $E$ is the temperature-dependent energy which is a source of the alteration. When $E$ is independent of temperature, we recover the standard form $d S=d Q / T$. This generalization asserts that the uncompensated heat in Clausius sense displaces the original entropy change of the system by an amount of $T^{-1}\langle d E / d T\rangle d T$.

We emphasize that the above background constitutes a sufficient incentive that must be addressed in a formulation of the second law equality on a sound footing: Heat and work that are exchanged/performed irreversibly should be quantified by combining temperature-dependent energy levels and the nonequilibrium work relation. The previous works do not cover the case of temperature-dependent energy levels. Therefore, the further extended rectification or improvement of the second law expression is possible. 


\section{Note on the terminology for the Clausius' $N$ :}

The following terminology used in different disciplines denotes essentially the same conception.

- Uncompensated transformation (Clausius [36])

- Second law equality (Tolman and Fine [10])

- Entropy balance (Chemical \& Mechanical engineering [33-35,48])

- Entropy generation or production (Prigogine and others [38,39])

- Entropy deficit function $[40,41]$

\subsection{The rectification via nonequilibrium work relation}

In this section, we look a bit closer the approach presented in $[40,41]$ and we shall point out that a further extended form is possible along the same line when a system has the temperature-dependent energy levels [49]. Let $d$ indicate an infinitesimal change in thermodynamic quantities during processes when the symbol is put before them. With this symbol, in general, we do not make any distinction according to irreversibility, whereas the symbol $\delta$ usually denotes infinitesimal and reversible changes in quantities.

The dissipated (or dissipative) work $(d W)_{d}$ plays a central role and it is an energy that the system does work on surroundings, during which the temperature of the system is usually different from the surrounding's one. In an infinitesimal process, it is defined as the difference between work exchanged irreversibly $(d W)_{i r r}$ and the one reversibly $(d W)_{\text {rev }}[32,40,41]$,

$$
(d W)_{d}=(d W)_{i r r}-(d W)_{r e v} .
$$

We understand these quantities as being averaged over repeated performances under same constraints, which provide operationally measureable irreversible relations. We omit here the bracket symbols \langle\rangle for denoting average for simplicity. In terms of heat exchanged, it can also be expressed as $(d W)_{d}=$ $(d Q)_{\text {rev }}-(d Q)_{\text {irr }}$, where $(d Q)_{\text {rev }}$ and $(d Q)_{\text {irr }}$ denote the heat exchanged by following irreversible and reversible paths between given initial and final states, respectively. The quantities in irreversible changes do not coincide with the reversible counterparts, i.e., $(d W)_{i r r} \neq(d W)_{r e v}$ and $(d Q)_{i r r} \neq(d Q)_{\text {rev }}$, which is the origin of dissipation (entropy generation $S_{G}$ ). Therefore, the second law indicates

$$
\frac{(d W)_{d}}{T}=S_{G}
$$

The central concern in engineering is to evaluate $(d W)_{d}$ experimentally or numerically [50]. On the other hand, according to the nonequilibrium work relation presented by Jarzynski [53], we have an intriguing relation

$$
\left\langle e^{-\beta W}\right\rangle=e^{-\beta \Delta F},
$$

where $\beta^{-1}=k_{B} T$ as usual and $\Delta F$ is the Helmholtz free energy difference between the two equilibrium states. The quantity $\Delta F$ is equivalent to $(d W)_{\text {rev }}$. The total work $W$ performed during a process 
inherently contains irreversible and reversible contributions. A merit of this relation is that measuring work in irreversible processes (i.e., real situations) requires finite time whereas one needs infinite time to measure the corresponding reversible work $(d W)_{\text {rev }}$. Therefore, the transformation from the reversible to the irreversible work is practically useful. However, this relation is only valid for processes that starts and ends at states that are in isothermal contact with a heat reservoir. Within the scope of this validity, Ben-Amotz and Honig [40,41], under the assumption that fluctuations in the irreversible work are small compared to $k_{B} T$, has combined Eq. (27) with the work relation (by regarding $W$ as $(d W)_{\text {irr }}$ ) and has derived the following equality expression:

$$
\frac{(d W)_{d}}{T}=k_{B} \ln \left\langle e^{-\frac{(d W)_{i r r}}{k_{B} T}}\right\rangle+\frac{(d W)_{i r r}}{T} .
$$

This tells us that the dissipated work can be obtained in finite time. We once again note that we are omitting the brackets symbols for $(d W)_{d}$ and $(d W)_{i r r}$. The brackets are understood to denote taking the arithmetic mean over the number of performances after taking the statistical mean of the factor within the logarithm over possible states. When the system is in contact with a reservoir and the temperatures are different (say, $T$ and $T_{0}$ respectively), the above result is generalized as [40,41]

$$
S_{G}=\left(1-\frac{T}{T_{0}}\right) \frac{(d Q)_{i r r}}{T}+\frac{(d W)_{d}}{T} .
$$

which is consistent in terms of positivity; the first term of the right hand side is positive because the heat flows from higher to lower temperatures and the second due to the positivity of dissipated work. This formulation can be applicable to the evaluation of entropy produced in a thermoelectric device, where an interface between two thermoelectric materials at different temperatures is exposed to a series of irreversible processes.

\subsection{Note for systems with temperature-dependent energy levels}

A further extension is in principle almost straightforward in cases where the materials have temperature-dependent energy levels. Consider now two systems 1 and 2, each of which has initially well-defined homogeneous temperatures $T_{1}$ and $T_{2}$, respectively. The heat flow between 1 and 2 occurs upon contact, and work is in general also exchanged between them as a result of mechanically irreversible motion. In this note, however, we exclude mechanical works because it is zero when thermoelectric devices are held fixed by constraints: i.e., $d U^{(j)}=(d Q)_{\text {rev } / \text { irr }}^{(j)},(j=1,2)$. Therefore, the total change in entropy $(d S)^{\text {total }}$ for the composite system $1+2$ in a reversible and/or irreversible process reads

$$
(d S)^{\text {total }}=\sum_{j=1}^{2}\left(\frac{(d Q)_{\gamma}^{(j)}}{T_{j}}-\frac{1}{T_{j}}\left\langle\frac{d E_{j}\left(T_{j}\right)}{d T_{j}}\right\rangle d T_{j}\right)
$$

where the symbol $\gamma$ designates either rev or $\mathrm{irr}$. Since the two systems are thermally in contact with each other at both initial and final equilibrium states, the heat exchanged satisfies a relation $(\Varangle Q)_{\text {rev }}^{1}+(d Q)_{\text {rev }}^{2}=$ 0 if it is done reversibly. This means that the absorbed heat by 1 reversibly can all be attributed to 2 as a 
heat source and vice versa. Hence as usual the sum of the first terms of the above indicates the transfer of the reversible heat from the higher temperature to the lower one:

$$
\frac{(d Q)_{r e v}^{1}}{T_{1}}+\frac{(d Q)_{r e v}^{2}}{T_{2}}=\left(\frac{1}{T_{1}}-\frac{1}{T_{2}}\right)(đ Q)_{r e v}^{1} .
$$

Thus, the total entropy change can be expressed as

$$
(d S)^{\text {total }}=\left(\frac{1}{T_{1}}-\frac{1}{T_{2}}\right)(d Q)_{r e v}^{1}-\sum_{j=1}^{2} \frac{1}{T_{j}}\left\langle\frac{d E_{j}\left(T_{j}\right)}{d T_{j}}\right\rangle d T_{j},
$$

which shows that if a process is done in an isothermal way $\left(T_{1}=T_{2}\right)$, the entropy produced comes purely from the existence of the temperature-dependent energy levels. The corresponding formulation for non-infinitesimal processes can be obtained by labeling infinitesimal expressions as intermediate steps of small finite changes and summing over them.

\subsection{An example - heat engine}

A formulation of the second law which is suitably reflected by the effect of the temperature-dependent energy levels in materials operated in a thermodynamic environment is a new direction. We consider the improved second law formulation applied to a thermodynamic cycle in this section. Since the thermoelectric power cycle can be regarded as a heat engine with electrons serving as the working fluid that cannot outperform the Carnot, we shall see how the efficiency is formulated when the temperature dependent energy effect is included. In an irreversible process, the entropy change is expressed as

$$
d S=\frac{(d Q)_{i r r}}{T}+S_{G}
$$

where we have used the above notation instead of one in Eq. (25). When the energy levels do not depend on temperature, the entropy generation $S_{G}$ is $(d W)_{d} / T$ with the dissipated work. However, in the present case, it is replaced by a modified form

$$
S_{G}=\frac{(d W)_{d}}{T}-\frac{1}{T}\left\langle\frac{d E_{l}}{d T}\right\rangle d T
$$

when a system has temperature-dependent energy levels [54]. The derivative of $l$ th energy level $E_{l}$ of the system with respect to temperature involves and the brackets denote an ensemble average over all microstates. For a cycle, the balance equations for energy and entropy are respectively given as

$$
\left\{\begin{array}{l}
\left(d \tilde{Q}_{h}\right)_{i r r}+\left(d W_{h}\right)_{i r r}+\left(d \tilde{Q}_{c}\right)_{i r r}+\left(d W_{c}\right)_{i r r}=0 \\
\frac{\left(d \tilde{Q}_{h}\right)_{i r r}}{T_{h}}+\frac{\left(d \tilde{Q}_{c}\right)_{i r r}}{T_{c}}+S_{G}=0 .
\end{array}\right.
$$

The first equation comes from the change of the internal energy vanishes $d U=0$ for a cycle. The second equation follows from the fundamental balance equation Eq. (34). Note however that the heat with the tilde symbol is clearly distinguished from the heat without it; i.e., $d \tilde{Q} \neq d Q$. The effective heat $d \tilde{Q}[46,47]$ must be used for the consistent thermodynamics description of the present case, which is given as $(d Q)_{i r r}-\left\langle\frac{d E_{l}}{d T}\right\rangle d T$. The suffixes $h$ and $c$ is to denote hot and cold, respectively. Therefore, irreversible works done from the higher and the lower temperature sources are denoted as $\left(d W_{h}\right)_{i r r}$ 
and $\left(d W_{c}\right)_{\text {irr }}$, respectively. Thus, the total work that the thermoelectric system gives to the sources is $-(d W)_{i r r}=-\left(d W_{h}\right)_{i r r}-\left(d W_{c}\right)_{i r r}$, and from Eq. (36) it can be expressed as

$$
-(d W)_{i r r}=\left(d \tilde{Q}_{h}\right)_{i r r}\left(1-\frac{T_{c}}{T_{h}}\right)-T_{c} S_{G} .
$$

If the cycle is done reversibly, the entropy generation vanishes $S_{G}=0$ and the suffixes read irr $\rightarrow$ rev. In this case, we have of course the usual Carnot form; $(d W)_{\text {rev }}=\left(d Q_{h}\right)_{\text {rev }}\left(1-T_{c} / T_{h}\right)$, in which $\left(d Q_{h}\right)_{\text {rev }}$ is heat reversibly transferred out of the higher temperature source. We have therefore the thermal efficiency $\eta$ for this engine defined as the ratio of the actual work and the invested effective heat:

$$
\begin{aligned}
\eta=\frac{-(d W)_{i r r}}{\left(d \tilde{Q}_{h}\right)_{i r r}} & =1-\frac{T_{c}}{T_{h}}-\frac{T_{c} S_{G}}{\left(d \tilde{Q}_{h}\right)_{i r r}} \\
& =1-\frac{T_{c}}{T_{h}}-\frac{T_{c} S_{G}}{\left(d Q_{h}\right)_{i r r}-\left\langle\frac{d E_{l}}{d T}\right\rangle(d T)_{h}},
\end{aligned}
$$

where $(d T)_{h}$ is a temperature difference induced by heat transfer at the boundary of the higher temperature side. Since the Carnot cycle has maximum in the efficiency among all types, this expression indicates that a relation $\left(d Q_{h}\right)_{i r r} /(d T)_{h}>\left\langle\frac{d E_{l}}{d T}\right\rangle$ has to be satisfied. In addition to the heat engine, the effects of the temperature-dependent energy levels on the performance characteristics for the heat transfer and the heat pump were investigated in Ref. ([54]), where the modified forms of efficiencies are derived. In conclusion, when one correctly includes the temperature dependency in energy levels in the description of entropy generation in thermoelectric device materials, new efficiency may be formulated.

\section{Summary}

This article showed an improved approach to the second law of thermodynamics for applications of thermoelectric systems. The new possibility is to take the existence of temperature-dependent energy levels of materials into consideration together with the associated thermodynamic treatment. This may potentially open up a direction for increasing the performance of devices, because the thermoelectric devices has long been confronted with difficulty in increase of their efficiencies. As we have focused, this issue may be improved from a different view point; irreversibility analysis or entropy production by considering the temperature dependency of the materials to optimize the design and the performance. The line of consideration presented will have more importance than ever when the size of the system gets smaller and the domain of temperature for the operation get down to $m K$ region. The author expects that this short overview enables to lead a better engineering of thermoelectric devices.

\section{Acknowledgements}

The author thanks Dawn E. Holmes (Conference Chair, University of California Santa Barbara) and Jely He (Managing Editor, Entropy) for the invitation to this conference as a section chair (Physics and engineering). Section 3 is based on the discussion with Professor Peter T. Landsberg (who passed away in February 2010 and put forth the approach with the general ensemble theory). The author thanks for hospitality during his stay in Southampton in November 2004. 


\section{Conflicts of Interest}

The author declares no conflict of interest.

\section{References}

1. A. Jäger-Waldau, Introduction to the topic and overview of existing scenarios for a sustainable/renewable energy supply, the presentation talk at the Symposium to Celebrate the 20th Anniversary of the German JSPS Alumni Association, "The Role of Renewable Energy for a Sustainable Energy Supply", held at Meiji University, October 6th, 2015.

2. Bell, L.E., Cooling, heating, generating power, and recovering waste heat with thermoelectric systems, Science, 321 (5895), (2008) 1457-1461.

3. Goldsmid, H. J., Introduction to Thermoelectricity, (Springer, 2010).

4. Callen, H.B., Thermodynamics and an introduction to thermostatistics 2nd ed. (New York: John Wiley\& Sons, 1985), Sect. 14.5-14.9.

5. Seebeck, T.J. Über den Magnetismus der galvenische Kette, Technical report, Reports of the Royal Prussian Academy of Science, Berlin, Germany, 1821.

6. Peltier, J.C.A. Nouvelles expériences sur la caloricité des courants électrique. Annales de Chimie et de Physique, 56, (1834) 371-386.

7. Thomson, W. On the Dynamical Theory of Heat, Trans. R. Soc. Edinburgh: Earth Sci., 3, (1851) 91-98.

8. Joffe, A.F. The revival of thermoelectricity, Sci. Am., 199, (1958) 31-37.

9. Goupil, C., Seifert, W., Zabrocki, K., Müller, E., Snyder, G.J., Thermodynamics of thermoelectric phenomena and applications, Entropy 13, (2011) 1481-1516.

10. Tolman, R.C., Fine, P.C., On the Irreversible Production of Entropy, Rev. Mod. Phys. 20, (1948) 51-77.

11. Phillips, J.C., Bonds and Bands in Semiconductors, Material Science series, pp. 150-152, Academic Press, New York (1973).

12. Varshni, Y.P. Temperature dependence of the energy gap in semiconductors, Physica 34 (1967) 149-154.

13. Cardona, M., Kremer, R.K., Temperature dependence of the electronic gaps of semiconductors, Thin Solid Films, 571, (2014), 680-683.

14. Sze, S.M., Physics of semiconductor devices, 2nd ed. (John Wiley \& Sons, NY. 1981).

15. G. S. Rushbrooke, On the statistical mechanics of assemblies whose energy-levels depend on the temperature, Trans. Faraday Soc. 36, (1940) 1055-1062.

16. A. Radkowsky, Temperature Dependence of Electron Energy Levels in Solids, Phys. Rev. 73, (1948) 749-761.

17. E. W. Elock and P. T. Landsberg, Temperature Dependent Energy Levels in Statistical Mechanics, Proc. Phys. Soc. B 70, (1957) 161-168.

18. de Miguel, R. Temperature-dependent energy levels and size-independent thermodynamics, Phys. Chem. Chem. Phys., 17, (2015) 15691-15693. 
19. Mott, N.F., Davis, E.A. Electronic Processes in Noncrystaline Materials (Oxford Classic Texts in the Physical Sciences), 2nd Ed. Sec. 7.4.5, Oxford University Press (2012).

20. Emin, D. Effect of temperature-dependent energy-level shifts on a semiconductor's Peltier heat, Phys. Rev. B 30, (1984) 5766-5770.

21. Emin, D. Reply to 'The effect of temperature-dependent energies on semiconductor thermopower formulae', Philos. Mag. B 51, (1985) L53-L56.

22. Emin, D. Effect of temperature-dependent band shifts on semiconductor transport properties, Solid State Commun. 22, (1977) 409-411.

23. Jeon, N. J., Noh, J. H., Yang, W. S., Kim, Y. C., Ryu, S., Seo, J. \& Seok, S. I., Compositional engineering of perovskite materials for high-performance solar cells, Nature 517, (2015) 476-480.

24. Foley, B. J., Marlowe, D. L., Sun, K., Saidi, W.A., Scudiero, L., Temperature dependent energy levels of methylammonium lead iodide perovskite, Appl. Phys. Lett. 106, (2015) 243904-5.

25. Landsberg,P. T., Yamano, T., The umbrella ensemble, unpublished work (2005).

26. Plastino, A., Plastino, A.R., On the universality of thermodynamics' Legendre transform structure, Phys. Lett. A 226 (1997) 257-263.

27. Yamano, T., On the robust thermodynamical structures against arbitrary entropy form and energy mean value, Eur. Phys. J. B 18, (2000) 103-106.

28. Landsberg, P. T., Thermodynamics with Quantum Statistical Illustrations, Sec.33, (New York, Interscience, 1960).

29. Landsberg, P. T., Foundation of Thermodynamics, Rev. Mod. Phys. 28 (1956) 363-392.

30. Giles, R., Mathematical foundation of thermodynamics, (Pergamon Press, 1964).

31. Lieb, E., and Yngvason, J., The physics and mathematics of the second law of thermodynamics, Physics Reports, 310, (1999) 1-96.

32. Kojima, K., Principle of efficient use of energy, Baifukan (2004) in Japanese.

33. Sandler, S.I., Chemical Engineering Thermodynamics, (3rd ed.), John Wiley \& Sons, Inc. (1999).

34. Wark, K. Jr., Richards, D.E., Thermodynamics (6th ed.), McGraw-Hill Education (ISE Editions) (1999).

35. Cengel, Y.A., Boles, M.A., Thermodynamics (in SI Units): An Engineering Approach, Chap. 6 (8th ed.), McGraw-Hill Education (2014).

36. Clausius, R., Mechanical theory of heat, (John van Voorst, 1867).

37. Clausius, R., Annalen der Physik und Chemie, Über eine veranderte Form des zweiten Hauptsatzes der mechanischen Wärmetheoriein, Bd. 93, (1854) 481-506.

38. D. Kondepudi, I. Prigogine, Modern Thermodynamics, From heat engines to dissipative structures, (John Wiley \& Sons, 1998).

39. I. Prigogine, Introduction to Thermodynamics of Irreversible Processes (Interscience Publishers, New York, 1955).

40. Ben-Amotz, D., Honig, J.M., The Rectified Second Law of Thermodynamics, J. Phys. Chem. B 110 (40), (2006) 19966-19972.

41. Ben-Amotz, D., Honig, J.M., Average Entropy Dissipation in Irreversible Mesoscopic Processes, Phys. Rev. Lett. 96, (2006) 020602-4.

42. Honig, J., On the Entropy of a Class of Irreversible Processes, Entropy 15, (2013) 2975-2988. 
43. J. Liphardt, S. Dumount, S. Smith, I. Tinoco, C. Bustamante, Sience, Equilibrium Information from Nonequilibrium Measurements in an Experimental Test of Jarzynski's Equality, 296, 1832-1835 (2002).

44. D. Collin, F. Ritort, C. Jarzynski, S. Smith, I. Tinoco, C. Bustamante, Nature, Verification of the Crooks fluctuation theorem and recovery of RNA folding free energies, 437, 231-234 (2005).

45. C. Bustamante, J. Liphardt, F. Ritort, Phys. Today, The Nonequilibrium Thermodynamics of Small Systems, 58, 43-48 (2005).

46. O. Shental and I. Kanter, Shannon meets Carnot: Generalized second thermodynamic law, EPL, 85, (2009) 10006-p5.

47. Peleg, Y., Efraim, H., Shental, O., Kanter, I., Mutual information via thermodynamics: Three different approaches, J. Stat. Mech. (2010) P01014.

48. Bejan, A., Entropy Generation Minimization, Chapter 7 (CRC Press: Boca Raton, FL, USA, 1996).

49. Yamano, T. Extended form of the second law equality for systems with temperature-dependent energy, presentation at the Joint European Thermodynamics Conference (JECT 2015) held at Nancy, France May 20-22, 2015.

50. The dissipated work multiplied by the ambient temperature is usually used in engineering thermodynamics [48]. It equals energy lost due to irreversibility and this relation is referred to as the Gouy-Stodola theorem [51,52].

51. Gouy, G., Journal de Physique, Sur l'energie utilizable, 8 (1889) 501-518.

52. Stodola, A., Steam and Gas Turbines, (McGraw-Hill, 1910).

53. Jarzynski, C., Phys. Rev. Lett. Nonequilibrium equality for free energy differences, 78 (1997) 2690-2693.

54. Yamano, T., Effect of temperature-dependent energy on entropy generation and its application to efficiencies, in preparation.

(c) 2015 by the author; licensee MDPI, Basel, Switzerland. This article is an open access article distributed under the terms and conditions of the Creative Commons Attribution license (http://creativecommons.org/licenses/by/3.0/). 